\title{
Estado actual de la Colección de Palmas (Arecaceae) del Herbario Nacional de Venezuela (VEN)
}

\section{Current status of the Palm collection (Arecaceae) of the Venezuelan National Herbarium (VEN) Yaroslavi Espinoza Flores}

Fundación Instituto Botánico de Venezuela, Gerencia de Investigación y Desarrollo - Herbario Nacional de Venezuela (VEN), Av. Salvador Allende, entrada Tamanaco UCV - Jardín Botánico de Caracas, Apartado Postal 2156, Caracas 1010-A, Venezuela.

Email: yespinoz@yahoo.com / yespinoz04@gmail.com
Trabajo presentado al Simposio Internacional "LAS PALMERAS EN EL Internacional LAS PALMERAS EN EL MARCO DE LA INVESTIGACION PARA EL DESARROLLO EN AMERICA DEL SUR, del 07 al 09 de Noviembre 2007 Museo de Historia Natural, Universidad Nacional Mayor de San Marcos, Lima, Perú.

\section{Resumen}

El Herbario Nacional de Venezuela (VEN) posee la mayor colección de muestras botánicas en el país, siendo de esta manera la principal fuente de referencia de la Flora de Venezuela, teniendo también un gran número de muestras de otros países del Neotrópico. El Herbario VEN contiene aproximadamente 400000 especimenes, de los cuáles 2192 constituyen la colección de palmas (Arecaceae), agrupados en 35 géneros (incluidos 5 géneros foráneos) y 127 taxa específicos e infraespecíficos, actualmente registrados en la base de datos del herbario. Los géneros con mayor número de especímenes son Geonoma (498) y Bactris (360), seguidos por el género Euterpe (158). La especie Geonoma deversa (Poit.) Kunth es la que contiene mayor número de especímenes dentro de la colección (110). El herbario cuenta con 12 especímenes tipos de la familia Arecaceae, incluyendo 4 holotipos, 1 lectotipo, 5 isotipos y 2 isoneotipos. El material ha sido determinado en su mayoría por especialistas como A. Henderson, F. Kahn, F. Stauffer y J. Wessels-Boer, entre otros. Se presenta una lista de las especies que se hallan depositadas en el herbario. De la revisión de los datos se obtuvo que los principales estados de Venezuela donde se han colectado palmas son: Amazonas, Bolívar, Zulia, Delta Amacuro, Aragua, Apure, y Distrito Capital. La contribución principal a la colección de palmas del herbario se debe a las recolecciones realizadas por J. A. Steyermark y por F. Stauffer.

Palabras clave: Arecaceae, Herbario VEN, Colección de palmas, Venezuela.

\section{Abstract}

The National Herbarium of Venezuela (VEN) has the largest collection of botanical specimens in the country, thus being the main source of reference for the flora of Venezuela. It also hosts a large number of specimens from other countries in the Neotropical region. The VEN contains approximately 400000 specimens currently registered in the herbarium's database, of which 2192 form the palm (Arecaceae) collection that encompasses 35 genera (including 5 foreign genera) and 127 specific and infraspecific taxa. The genera with the largest number of specimens are Geonoma (498) and Bactris (360), followed by Euterpe (158). The species Geonoma deversa (Poit.) Kunth contains the largest number of specimens in the collection (110). The herbarium hosts type material of 12 Arecaceae taxa: 4 holotypes, 1 lectotype, 5 isotypes and 2 isoneotypes. Most of the specimens at the VEN have been identified by specialists such as A. Henderson, F. Kahn, F. Stauffer and J. WesselsBoer. A list of species found in the herbarium is presented herein. Review of specimen data at the VEN show that palms have been collected mainly in the following states of Venezuela: Amazonas, Bolivar, Zulia, Delta Amacuro, Aragua, Apure, and Distrito Capital. The main contribution to the collection of palms to the VEN is the collections made by J. A. Steyermark and F. Stauffer.

Keywords: Arecaceae, VEN Herbarium, palm collection, Venezuela

\section{Introducción}

El Herbario Nacional de Venezuela (VEN), se fundó en 1921 como parte del Museo Comercial e Industrial de Venezuela, el cual estaba bajo la dirección del naturalista Henri Pittier. Se inició con colecciones de José María Vargas, Adolfo Ernst, Alfredo Jahn y del propio fundador. La sección botánica del Museo fue transferida luego al servicio botánico del Ministerio de Agricultura y Cría, el cual se fundó en 1936. En 1958, fue trasladado desde el Museo a su sede actual en el Jardín Botánico de Caracas. Desde 1991 está adscrito a la Fundación Instituto Botánico de Venezuela (FIBV), ubicada dentro de la Ciudad Universitaria de Caracas, decretada por la UNESCO como patrimonio de la humanidad en el año 2000. El Herbario posee la mayor colección de muestras botánicas en el país, siendo de esta manera la principal fuente de referencia de la Flora de Venezuela, teniendo también un gran número de muestras de otros países del Neotrópico, contiene aproximadamente 400.000 especímenes o muestras. (Http://www.fibj.org.ve; Rodríguez y Hokche, 2006).

El objetivo principal de éste trabajo es dar a conocer los resultados de la revisión realizada en la colección de las palmas (Arecaceae) en el herbario VEN, como inicio al estudio del estado actual de la familia en Venezuela.

\section{Material y métodos}

La información de la colección, se extrajo de la revisión de los especímenes y fue corroborada con la información de la base de datos del Herbario; se tomaron en cuenta principalmente datos sobre: el número de géneros, de especies, de especímenes o muestras por especies, localidades, colectores principales, especialistas que han revisado la colección y las muestras Tipo de palmas.

Los especímenes estudiados se encuentran organizados en el herbario en orden alfabético de familia, géneros y especies, dentro de los estantes, ubicados en carpetas y en cajas que resguardan el material tanto vegetativo como reproductivo, en algunos casos el material dentro de las cajas se encuentra montado en láminas, pero en la mayoría de las cajas el material se encuentra sin montar.

El material ha sido determinado principalmente por especialistas como Andrew Henderson, Francis Kahn, Fred Stauffer, Jan Gerard Wessels-Boer, entre otros.

\section{Resultados y Discusión Riqueza de la colección}

En el Herbario VEN existen 35 géneros (incluidos 05 géneros foráneos) y 127 taxa específicos e infraespecíficos, distribuidos en 
2192 especímenes de palmas (Arecaceae) actualmente registrados en la base de datos del herbario.

Las especies de palmas existentes en el herbario VEN se presentan en la tabla 1, con su respectivo número de especímenes. Los nombres de las especies resaltadas en negritas, se refieren a las determinaciones que deben ser revisadas, para cambios de sinonimia, nombres dudosos, especies extranjeras, indeterminadas, y malas determinaciones. Del total de los 2192 especímenes de palmas, 123 están indeterminados y 190 se encuentran determinados hasta género.

En total existen 313 muestras botánicas por determinar hasta especie, lo que representa un $14 \%$ dentro de la colección de palmas.

Los géneros con mayor número de especímenes son Geonoma (498) y Bactris (360), seguidos por el género Euterpe (158). La especie Geonoma deversa (110) es la que contiene mayor número de especímenes.

\section{Distribución geográfica en Venezuela}

De la revisión de la colección del herbario se obtuvo que los principales estados de Venezuela donde se han colectado palmas son: Amazonas, Bolívar, Zulia, Aragua, Apure, Distrito Capital, Yaracuy, y Miranda, entre otros (Mapa, figura 1).

Estos datos son similares a lo reportado por Stauffer en (1999), donde Amazonas y Bolívar aparecen como los principales estados con mayor cantidad de especies colectadas. Stauffer, hace énfasis en su trabajo en que los datos obtenidos estaban influenciados por los esfuerzos de colección y la gran cantidad de estudios realizados en ambos estados venezolanos.

Esto nos puede dar una idea de las localidades y/o zonas del país que requieren mayor atención en cuanto al estudio de las palmas a nivel taxonómico; y donde realizar mayor número de

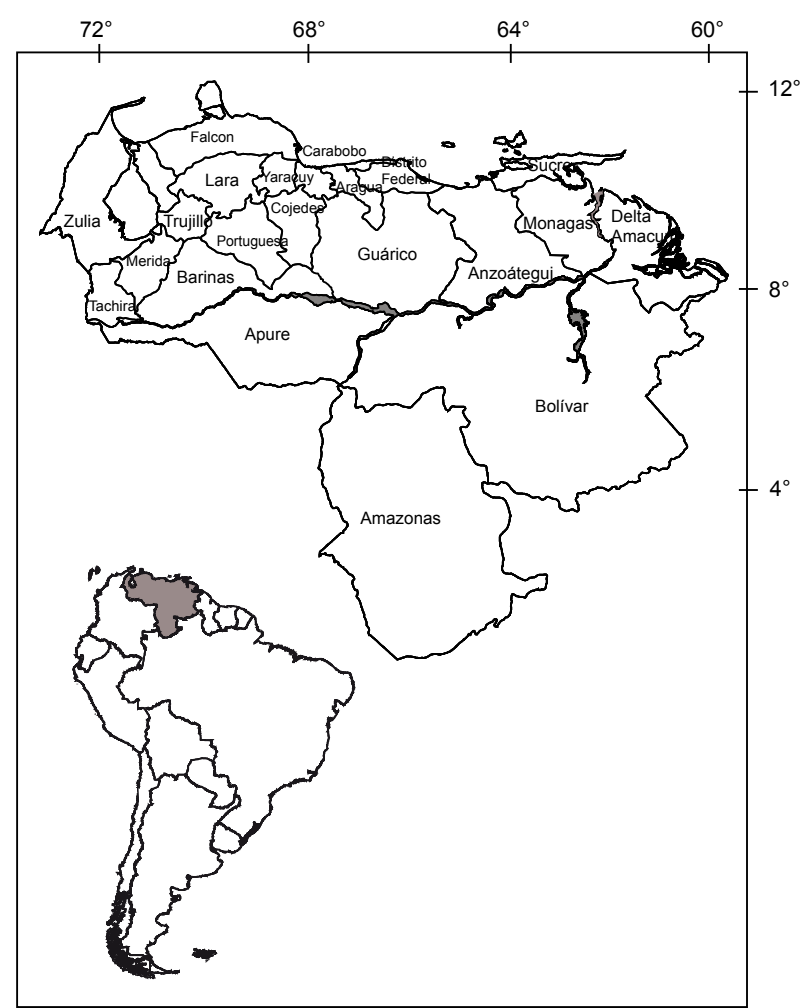

Figura 1. Mapa político de Venezuela y su ubicación en Sudamérica.
Tabla 1. Lista de las especies de palmas (Arecaceae) en el Herbario VEN y número de especímenes o muestras botánicas.

\begin{tabular}{|c|c|}
\hline Lista de Especies & $\begin{array}{c}\mathrm{N} .^{\circ} \text { de } \\
\text { muestras por } \\
\text { especie }\end{array}$ \\
\hline 1. Acrocomia aculeata & 8 \\
\hline 2. Aiphanes aculeata & 8 \\
\hline 3. Arenga caudata & 1 \\
\hline 4. Asterogyne ramosa & 5 \\
\hline 5. Asterogyne spicata & 15 \\
\hline 6. Asterogyne yaracuyense & 8 \\
\hline 7. Astrocaryum acaule & 18 \\
\hline 8. Astrocaryum aculeatum & 3 \\
\hline 9. Astrocaryum chambira & 2 \\
\hline 10. Astrocaryum gynacanthum & 58 \\
\hline 11. Astrocaryum jauari & 27 \\
\hline 12. Astrocaryum murumuru var. murumuru & 1 \\
\hline 13. Astrocaryum sp. & 6 \\
\hline 14. Attalea butyracea & 9 \\
\hline 15. Attalea maripa & 23 \\
\hline 16. Attalea microcarpa & 8 \\
\hline 17. Attalea racemosa & 21 \\
\hline 18. Attalea sp. & 2 \\
\hline 19. Bactris acanthocarpa var. acanthocarpa & 19 \\
\hline 20. Bactris balanophora & 7 \\
\hline 21. Bactris bidentula & 7 \\
\hline 22. Bactris brongniartii & 16 \\
\hline 23. Bactris campestris & 3 \\
\hline 24. Bactris corossilla & 29 \\
\hline 25. Bactris elegans & 1 \\
\hline 26. Bactris gasipaes & 1 \\
\hline 27. Bactris gasipaes var. chichagui & 9 \\
\hline 28. Bactris gasipaes var. gasipaes & 3 \\
\hline 29. Bactris guineensis & 23 \\
\hline 30. Bactris hirta & 7 \\
\hline 31. Bactris hirta var. hirta & 19 \\
\hline 32. Bactris hirta var. pectinata & 12 \\
\hline 33. Bactris major & 14 \\
\hline 34. Bactris major var. infesta & 1 \\
\hline 35. Bactris major var. major & 2 \\
\hline 36. Bactris maraja & 8 \\
\hline 37. Bactris maraja var. maraja & 25 \\
\hline 38. Bactris maraja var. trichospatha & 1 \\
\hline 39. Bactris oligoclada & 4 \\
\hline 40. Bactris pilosa & 7 \\
\hline 41. Bactris ptariana & 7 \\
\hline 42. Bactris setulosa & 27 \\
\hline 43. Bactris simplicifrons & 79 \\
\hline 44. Bactris sp. & 29 \\
\hline 45. Barcella odora & 1 \\
\hline 46. Caryota urens & 1 \\
\hline 47. Ceroxylon ceriferum & 4 \\
\hline
\end{tabular}




\section{Tabla 1. Continuación}

Lista de Especies
N. ${ }^{\circ}$ de

muestras por especie
48. Ceroxylon interruptum

49. Ceroxylon $\mathrm{sp}$.

50. Chamaedorea linearis

51. Chamaedorea pinnatifrons

52. Chamaedorea sp.

53. Chamaedorea tepejilote

54. Coccothrinax barbadensis

55. Cocos nucifera

56. Copernicia tectorum

57. Cryosophila warscewiczii

58. Cryosophila guaguara

59. Desmoncus mitis var. mitis

60. Desmoncus orthacanthos

61. Desmoncus phoenicocarpus

62. Desmoncus polyacanthos var. polyacanthos

63. Desmoncus sp.

64. Dictyocaryum fuscum

65. Dictyocaryum ptarianum

66. Dictyocaryum sp.

67. Euterpe catinga var. catinga

68. Euterpe catinga var. roraimae

69. Euterpe edulis

70. Euterpe longibracteata

71. Euterpe longipetiolata

72. Euterpe oleracea

73. Euterpe precatoria

74. Euterpe precatoria var. longevaginata

75. Euterpe precatoria var. precatoria

76. Euterpe sp.

77. Geonoma appuniana

78. Geonoma aspidiifolia

79. Geonoma baculifera

80. Geonoma cuneata

81. Geonoma densa

82. Geonoma deversa

83. Geonoma gracilis

84. Geonoma interrupta

85. Geonoma interrupta var. interrupta

86. Geonoma jussieuana

87. Geonoma leptospadix

88. Geonoma macrostachys var. acaulis

89. Geonoma macrostachys var. poiteauana

90. Geonoma maxima

91. Geonoma maxima var. ambigua

92. Geonoma maxima var. maxima

93. Geonoma orbignyana

94. Geonoma paraguanensis

95. Geonoma simplicifrons

\section{Tabla 1. Continuación}

Lista de Especies

N. ${ }^{\circ}$ de

muestras por especie

96. Geonoma sp. $\quad 75$

97. Geonoma spinescens 4

98. Geonoma spinescens var. braunii 13

99. Geonoma spinescens var. spinescens 13

100. Geonoma stricta var. stricta 3

101. Geonoma undata 32

102. Geonoma weberbaueri 6

103. Hyospathe elegans 31

104. Iriartea deltoidea

105. Iriartella setigera $\quad 88$

106. Leopoldinia major $\quad 4$

107. Leopoldinia piassaba 10

108. Leopoldinia pulchra 39

109. Leopoldinia sp. 3

110. Lepidocaryum tenue var. casiquiarense 12

111. Manicaria saccifera 20

112. Mauritia carana 4

113. Mauritia flexuosa 19

114. Mauritia sp. $\quad 8$

115. Mauritiella aculeata $\quad 29$

116. Mauritiella armata $\quad 12$

117. Mauritiella sp. $\quad 1$

118. Oenocarpus bacaba 22

119. Oenocarpus balickii $\quad 4$

120. Oenocarpus bataua 5

121. Oenocarpus bataua var. bataua 22

122. Oenocarpus bataua var. oligocarpa 8

123. Oenocarpus mapora $\quad 6$

124. Oenocarpus minor 1

125. Oenocarpus sp. 8

126. Prestoea acuminata 13

127. Prestoea acuminata var. acuminata $\quad 15$

128. Prestoea carderi 11

129. Prestoea longepetiolata var. cuatrecasasii 1

130. Prestoea pubigera 15

131. Prestoea sp. 3

132. Prestoea tenuiramosa 6

133. Roystonea oleracea var. oleracea 11

134. Sabal mauritiiformis $\quad 11$

135. Scheelea wesselsboeri $\quad 1$

136. Socratea exorrhiza 54

137. Socratea pittierana 1

138. Syagrus orinocensis $\quad 14$

139. Syagrus sancona $\quad 7$

140. Syagrus $s p$.

141. Syagrus stenopetala 9

142. Wettinia praemorsa 41 
Tabla 2. Número de especies por Estado, basado en datos del Herbario VEN.

\begin{tabular}{|c|c|}
\hline Estados & N..$^{\circ}$ de especies \\
\hline Amazonas & 68 \\
\hline Anzoátegui & 7 \\
\hline Apure & 19 \\
\hline Aragua & 19 \\
\hline Aragua - Miranda & 2 \\
\hline Barinas & 12 \\
\hline Bolívar & 50 \\
\hline Carabobo & 12 \\
\hline Carabobo - Aragua & 1 \\
\hline Cojedes & 12 \\
\hline Delta Amacuro & 22 \\
\hline Distrito Capital & 18 \\
\hline Distrito Capital - Aragua & 3 \\
\hline Distrito Capital -Miranda & 4 \\
\hline Falcón & 12 \\
\hline Falcón - Lara & 2 \\
\hline Guárico & 5 \\
\hline Guárico - Miranda & 3 \\
\hline Lara & 14 \\
\hline Lara - Falcón & 1 \\
\hline Mérida & 9 \\
\hline Miranda & 16 \\
\hline Miranda - Aragua & 1 \\
\hline Monagas & 15 \\
\hline Nueva Esparta & 5 \\
\hline Portuguesa & 5 \\
\hline Sucre & 16 \\
\hline Táchira & 15 \\
\hline Trujillo & 11 \\
\hline Vargas & 7 \\
\hline Yaracuy & 17 \\
\hline Zulia & 23 \\
\hline
\end{tabular}

recolección de material para herbario. En la tabla 2 se presenta la lista de la distribución de las especies de palmas según los datos obtenidos de los especímenes del herbario.

Entre los especialistas y colectores de palmas de los especímenes del Herbario VEN se destacan: J. Steyermark, F. Stauffer, R. Liesner, F. Delascio, G. Davidse, Ll. Williams, W. Meier, F. Guánchez, A. Henderson, F. Tamayo, H. Pittier, y A. Braun;

Tabla 3. Principales colectores de palmas de los especímenes depositados en el Herbario VEN.

\begin{tabular}{lcc} 
Colector & $N^{o}$ de especímenes & Fechas de Colección \\
\hline Steyermark, J. & 406 & $1944-1986$ \\
Stauffer, F. & 287 & $1991-2002$ \\
Liesner, R. & 172 & $1977-1988$ \\
Delascio, F. & 94 & $1979-2004$ \\
Davidse, G. & 90 & $1973-1984$ \\
Williams, Ll. & 81 & $1939-1946$ \\
Meier, W. & 67 & $1991-2001$ \\
Guánchez, F. & 48 & $1980-1984$ \\
Henderson, A. & 40 & $1984-1989$ \\
Tamayo, F. & 29 & $1939-1954$ \\
Pittier, H. & 20 & $1922-1947$ \\
Braun, A. & 14 & $1962-1979$ \\
\hline
\end{tabular}

entre otros colectores cuyo aporte a enriquecido la Colección de Palmas del Herbario VEN (Tabla 3). La contribución principal se debe a los aportes realizados por el Dr. Julián Steyermark entre los ańos 1944 hasta 1986 con 406 especímenes, y por el Dr. Fred Stauffer con aproximadamente 287 especímenes desde 1991 hasta el 2002.

\section{Tipos de palmas (Arecaceae) en el Herbario VEN}

La colección de Tipos de palmas fue publicada por Stauffer \& Stauffer en (1996), luego de esta publicación a la colección ingresaron los isotipos de Aiphanes aculeata Willd y Prestoea acuminata (Willd.) H. E. Moore var. acuminata. A.J. Hend. \& Galeano, y también se depositó el holotipo de Geonoma spinescens var. braunii F.W. Stauffer.

A continuación se presenta la lista de los tipos revisados y sus publicaciones:

1. Aiphanes aculeata Willd. Borchsenius, F. y R. Bernal, Aiphanes (Palmae) Flora Neotropica. Monografía 70: 46. 1996. Isoneotipo (NY y VEN): Steyermark \& Espinoza 106916. (7 partes). =Aiphanes horrida (Jacq.) Burret, Notizbl. Bot. Gart. Berlin-Dahlem 11: 575 (1932).

2. Aristeyera ramosa H. E. Moore, J. Arnold Arb. 48(3): 144.1967. Isotipo: J. Steyermark \& M. Rabe 96.088 (VEN!) (3 partes). =Asterogyne ramosa (H. E. Moore) Wess. Boer, Verh. Kon. ned. Akad. Wetensch., afd. Nat., 2 reeks, 58: 81. 1968.

3. Aristeyera spicata H. E. Moore, J. Arnold Arb. 47(1): 5-9. 1966. Isotipo: J. Steyermark 90.010 (VEN!) (3 partes). =Asterogyne spicata (H. E. Moore) Wess. Boer, Verh. Kon. Ned. Akad. Wetensch., afd. Nat., 2 reeks, 58: 82. 1968.

4. Asterogyne yaracuyense A. J. Hend. \& Steyerm., Brittonia 38(4): 309-313. 1986. Holotipo: J. Steyermark, G. Bunting \& J. G. Wessels-Boer 100.311 (VEN!) (3 partes).

5. Bactris bergantina Steyerm., Fieldiana Bot. 28(1): 71. 1951. Isotipo: J. Steyermark 61.039 (VEN!) (3 partes). =Bactris setulosa H. Karst. Linnaea 28: 408. 1857.

6. Chamaedorea minor Burret, Notizbl. Bot. Gart. BerlinDahlem, 11:2. 1930. Holotipo: J. Saer 427 (VEN!) (2 partes). =Chamaedorea pinnatifrons (Jacq.) Oerst., Vidensk. Medel. Dansk. Naturhist. Foren. kjoebenhavn 1858: 14.1859.

7. Geonoma tenuis Burret, Notizbl. Bot. Gart. BerlinDahlem, 13: 478. 1937. Isotipo: H. Pittier 13.840 (VEN!) (1 parte). =Geonoma spinescens $\mathrm{H}$. Wendl. ex Burret, Bot. Jahrb. 63:230. 1930.

8. Geonoma spinescens H. Wendl. var. braunii F. W. Stauffer., Acta Bot. Venez. 20(2): 5. 1997. Holotipo: Fernández, A. et al. 10087 (VEN!) (3 partes). =Geonoma spinescens. H. Wendl., Bot. Jahrb. Syst. 63: 230 (1930).

9. Hyospathe pittieri Burret, Notizbl. Bot. Gart. BerlinDahlem, 14:137. 1938. Lectotipo: H. Pittier 14.146 (VEN!)(1 parte). =Hyospathe elegans Mart., Hist. Nat. Palm. 2:1. 1823. 
10. Mauritia flexuosa var. venezuelana Steyerm., Fieldiana Bot. 28(!): 90. 1951. Isotipo: J. Steyermak 57.649 (VEN!) (1 parte). =Mauritia flexuosa. L.f. Suppl. 454. 1781.

11. Prestoea acuminata. (Willd.) H. E. Moore var. acuminata. Henderson, A. y G. Galeano, 1996. Euterpe, Prestoea y Neonicholsonia (Palmae). Flora Neotrópica. Monografía 72:48-49. 1996. Isoneotipo: J. Steyermark 91.608 (VEN!) (4 partes).

12. Syagrus stenopetala Burret, Notizbl. Bot. Gart. BerlinDahlem, 11:322. 1932. Holotipo: A. Jahn 1208 (VEN!) (2 partes). =Syagrus orinocensis (Spruce) Burret, Notizbl. Bot. Gart. Berlin-Dahlem, 13: 695. 1937.

\section{Conclusiones}

$\mathrm{Al}$ igual que lo encontrado por Stauffer (1999), se evidenció la gran necesidad de llevar a cabo un completo estudio de la familia en las áreas donde las colecciones son realmente escasas, ya que siguen existiendo deficiencias en algunos de los estados al norte del país, en zonas que se pudieran considerar tesoros o paraísos de palmas, como es el caso de Carabobo, Falcón, Lara, Miranda, Sucre, Yaracuy, y Zulia.

Igualmente se puede notar que se ha manteniendo una preferencia o "favoritismo" al momento de recolectar especímenes de individuos de hábito de porte bajo e inerme, por lo que el conocimiento taxonómico de especies autóctonas de porte alto o armadas, continua reducido en la mayoría de las especies de este tipo, por el escaso material que se encuentra en los herbarios. Y además se debe hacer un énfasis en estudios que abarquen no solamente la taxonomía y florística de la familia, sino que se tomen en cuenta estudios ecológicos, fitogeográficos, de conservación, y etnobotánicos, tomando en consideración aquellas zonas que presentan amenazas en sus ecosistemas o que han sido poco estudiadas.

\section{Agradecimientos}

A la Dra. Leyda Rodríguez por su orientación y apoyo en el manejo de la colección de palmas del herbario VEN; a la TSU. Maria de los Ángeles Castillo por su aporte en el registro de los especímenes de las palmas en la base de datos del herbario; al Dr. Fred Stauffer, el Dr. Aníbal Castillo, y al Lic. Rafael Ghinaglia por sus recomendaciones en la redacción de esta investigación; y finalmente al Lic. Héctor Pineda por su apoyo en la búsqueda de la bibliografía en la biblioteca "Henri Pittier" de esta Fundación.

\section{Literatura Citada}

Henderson, A., G. Galeano, \& R. Bernal. 1995. Field guide to the Palms of the Americas. Princeton University Press, Princeton, N. J. 353 pp.

Rodríguez, L. \& O. Hokche. 2006. Herbario Nacional de Venezuela (VEN): 85 años de Historia y Representación de la Flora Venezolana. Acta Bot. Venez. 29 (2): 363-368.

Stauffer, F. \& L. P. de Stauffer. 1996. Tipos de palmas (Arecaceae) del Herbario Nacional de Venezuela. Acta Bot. Venez. 19(1): 60-75.

Stauffer, F. 1999. Datos preliminares a la actualización de la flora de palmas (Arecaceae) de Venezuela. Acta Bot. Venez. 22(1): 77:107.

Stauffer, F. \& J. Fariñas. 2006. The identity of Attalea macrolepis (Burret) Wess. Boer (Arecaceae). Candollea 61(1): 8388. 


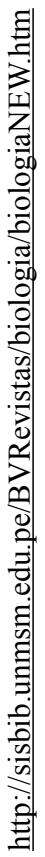

